Draft VERSION OCTOBER 16, 2015

Preprint typeset using LATEX style emulateapj v. 5/2/11

\title{
IMPLICATIONS FOR THE FORMATION OF BLUE STRAGGLER STARS FROM HST ULTRAVIOLET OBSERVATIONS OF NGC 188
}

\author{
Natalie M. Gosnell ${ }^{1,2}$, Robert D. Mathieu ${ }^{2}$, Aaron M. Geller $^{3,4}$, Alison Sills ${ }^{5}$, Nathan Leigh ${ }^{6,7}$, Christian $^{2}$ \\ KNIGGE $^{8}$ \\ Draft version October 16, 2015
}

\begin{abstract}
We present results of a Hubble Space Telescope far-ultraviolet (FUV) survey searching for white dwarf (WD) companions to blue straggler stars (BSSs) in open cluster NGC 188. The majority of NGC 188 BSSs (15 of 21) are single-lined binaries with properties suggestive of mass-transfer formation via Roche lobe overflow, specifically through an asymptotic giant branch star transferring mass to a main sequence secondary, yielding a BSS binary with a WD companion. In NGC 188, a BSS formed by this mechanism within the past 400 Myr will have a WD companion hot and luminous enough to be directly detected as a FUV photometric excess with HST. Comparing expected BSS FUV emission to observed photometry reveals four BSSs with WD companions above 12,000 K (younger than 250 Myr) and three WD companions with temperatures between 11,000-12,000 K. These BSS+WD binaries all formed through recent mass transfer. The location of the young BSSs in an optical color-magnitude diagram (CMD) indicates that distance from the zero-age main sequence does not necessarily correlate with BSS age. There is no clear CMD separation between mass transfer-formed BSSs and those likely formed through other mechanisms, such as collisions. The seven detected WD companions place a lower limit on the mass-transfer formation frequency of $33 \%$. We consider other possible formation mechanisms by comparing properties of the BSS population to theoretical predictions. We conclude that 14 BSS binaries likely formed from mass transfer, resulting in an inferred mass-transfer formation frequency of approximately $67 \%$.
\end{abstract}

\section{INTRODUCTION}

Open clusters are ideal environments for studying stellar populations. The ability to isolate a single-age stellar population from the field using radial-velocity $(\mathrm{RV})$ and proper-motion measurements not only informs our understanding of normal single-star evolution, but also highlights those stars whose evolutionary paths deviate from single-star expectations. One such group of objects is blue straggler stars (BSSs). Traditionally defined to be star cluster members that are brighter and bluer than the corresponding main sequence turnoff (Sandage 1953), the definition extends to stars that appear to be too young given the cluster age, such as stars below the turnoff but blueward of the main sequence (Mathieu \& Geller 2009). BSSs are not anomalous objects; they are found in open clusters (e.g. Johnson \& Sandage 1955; Burbidge \& Sandage 1958;

gosnell@astro.as.utexas.edu

${ }^{1}$ Department of Astronomy, The University of Texas at Austin, 2515 Speedway, Stop C1400, Austin, TX 78712-1205

2 Department of Astronomy, University of Wisconsin - Madison, 475 N. Charter Street, Madison, WI 53706

3 Center for Interdisciplinary Exploration and Research in Astrophysics (CIERA) and Department of Physics and Astronomy Northwestern University, 2145 Sheridan Rd, Evanston, IL 60208

${ }^{4}$ Department of Astronomy and Astrophysics, University of Chicago, 5640 S. Ellis Avenue, Chicago, IL 60637

${ }^{5}$ Department of Physics and Astronomy, McMaster University, 1280 Main St. W, Hamilton, ON L8S 4M1, Canada

6 Department of Physics, University of Alberta, CCIS 4-183, Edmonton, AB T6G 2E1, Canada

7 Department of Astrophysics, American Museum of Natural History, Central Park West and 79th Street, New York, NY 10024

8 School of Physics and Astronomy, University of Southampton, Highfield, Southampton, SO17 IBJ, UK
Leonard 1996; Sandquist 2005; Talamantes et al. 2010), globular clusters (e.g., Sandage 1953; Ferraro et al. 1999; Piotto et al. 2004; Leigh. Sills. \& Knigge 2007; Knigge et al. 2009; Santana et al. 2013), the Galactic field (Preston \& Sneden 2000; Carnev et al. 2001), and dwarf galaxies (Momany et al. 2007; Mapelli et al.|2007). The BSS population of open cluster NGC 188, in addition to a few sub-subgiants and yellow giants, comprises approximately $25 \%$ of the evolved cluster population (Mathieu \& Geller 2014, and references therein). Thus, understanding the creation and subsequent evolution of BSSs and other non-standard stellar products is fundamental to completing the full picture of stellar evolution at large.

BSSs were first discovered about 60 years ago (Sandage 1953; Johnson \& Sandage 1955), and understanding their origin continues to be an active field of research. A common theme is that BSS progenitors must gain additional mass in order to appear above the main sequence turnoff, but theories differ on how the mass is acquired. There are currently three primary theories for binary BSS formation: stellar collisions during dynamical interactions of binaries with single stars or other binaries (e.g., Leonard 1989; Ferraro et al. 1995; Mapelli et al. 2006; Leigh \& Sills 2011), the merger of an inner binary in a triple system via the Kozai mechanism (Perets \& Fabrycky 2009; Naoz \& Fabrycky 2014), and Roche lobe overflow mass transfer while the original (progenitor) primary star is on the red giant branch (RGB, Case B) or asymptotic giant branch (AGB, Case C; e.g., McCrea 1964; Ferraro et al. 2001, 2006; Chen \& Han 2008; Leigh. Sills, \& Knigge 2011; Leigh et al. 2013; Gosnell et al. 2014). Although not a likely pathway for binary BSSs, mass transfer while both 
stars are on the main sequence (Case A) can create single BSSs (Webbink 1976; ;ombardi et al. 2002).

Broadly, all these formation scenarios create BSSs similar to those observed in star clusters, but the details of the BSS population will vary. Collisions can create single BSSs or binary BSSs that retain a companion from the dynamical encounter (e.g., Hurley et al. 2005; Leigh \& Sills 2011; Leigh \& Geller 2012). (In this work, a collision product is a star resulting from a collision, coalescence, or merger of two stars during a dynamical encounter.) BSSs formed through Case A mass transfer typically lack a binary companion (Webbink 1976; Lombardi et al. 2002), although Tian et al. (2006) model the formation of BSSs from Case A mass transfer that result in binaries with periods less than 10 days. The Kozai mechanism will create a binary BSS whose secondary is the original tertiary member of the system (Perets \& Fabrycky 2009). A BSS formed from Roche lobe overflow will also be in a binary, but the secondary star will be the core of the progenitor primary star, observed as a carbon/oxygen (CO) or helium white dwarf (WD; Geller \& Mathieu 2011; Geller et al. 2013; Gosnell et al. 2014).

The number of BSSs observed in a star cluster is determined by a combination of the formation rate for a given mechanism and the corresponding BSS lifetime. $N$-body models are capable of predicting the number of BSSs in cluster environments created through each formation method (Hurley et al. 2005; Geller et al. 2013). However, empirical determination of BSS formation for a given population is necessary in order to make appropriate comparisons to $N$-body model results.

Observational identification of the particular formation mechanisms for an entire population of BSSs has proved elusive, although formation pathways for several individual BSSs have been determined in globular clusters. Knigge et al. (2008) find a globular cluster BSS+WD binary that perhaps formed through mass transfer (although it may also be the result of a dynamical exchange) and Rozyczka et al. (2013) find a globular cluster BSS currently undergoing mass transfer. In 47 Tuc, Ferraro et al. (2006) discover five BSSs with depleted CO that is suggestive of a mass transfer history and Knigge et al. (2006) find a BSS likely formed from at least three stars, but (by design) none of these studies provide enough context to learn about formation mechanisms of the entire BSS population. In globular cluster M30, Ferraro et al. (2009) find a trend in optical color and magnitude that suggest two BSS subpopulations split between mass transfer- and collisionformed products, but further observational confirmation, such as detecting BSS binarity, is very difficult due to the crowded nature of globular clusters. Finally, the existence of W UMa-type binaries in the BSS region of globular clusters (e.g., Rucinski 2000) is evidence that mass transfer must be responsible for at least a portion of globular cluster BSS populations.

In an attempt to disentangle the problem of multiple formation pathways we focus on the population of BSSs in open cluster NGC 188, which presents an excellent environment for studying these objects. The cluster is one of the oldest known open clusters in the Galaxy, with an age of 7 Gyr (Sarajedini et al. 1999). The BSSs, therefore, are not very "blue," allowing for accu- rate RV measurements and binary orbit determinations (Geller et al. 2008, 2009). NGC 188 is also relatively close (1770 \pm 75 pc; Meibom et al. 2009) so observations from both ground and space are not prohibitively expensive. The WIYN Open Cluster Study Mathieu 2000) has observed NGC 188 for almost 20 years, obtaining photometry (Sarajedini et al. 1999), proper motions (Platais et al. 2003), RVs (Geller et al. 2008), and binary orbits (Geller et al. 2009), and has expanded into theoretical efforts with sophisticated $N$-body models covering the entire cluster lifetime (Geller et al. 2013). This enormous foundation of data makes the NGC 188 BSS population one of the most well-studied in the Galaxy.

The NGC 188 BSS population includes 21 stars, 20 of which are high-probability 3-dimensional cluster members (Platais et al. 2003; Geller et al. 2008). The single exception, WOCS 4230 , is a binary star photometrically classified as a BSS but lacks a solved binary orbit due to rapid rotation, resulting in an unknown RV-determined cluster membership. (Platais et al. 2003 determine a proper motion membership probability for WOCS 4230 of 93\%.) The majority (80\%) of the BSSs in NGC 188 are spectroscopic binaries (Mathieu \& Geller 2009). Four BSSs do not have detected RV variability, meaning they are not in binaries with orbital periods less than $10^{4}$ days (Geller et al. 2009). They either have companions at very long periods, $10^{4}<P_{\text {orb }}<10^{6}$ days, or are single stars (Geller \& Mathieu 2012). Binaries with periods beyond $10^{6}$ days are thought to be destroyed through dynamical interactions in the cluster environment. The BSS population includes two double-lined (SB2) binaries with very short orbital periods around 5 days and high mass ratios of $q=1.0$ and $q=0.68$ (Geller et al. 2009). The remaining 15 BSS binaries are single-lined, which we refer to as SB1 systems. In Table 1 we present the NGC 188 BSS population. We list the identification number (WOCS ID), J2000 location, optical photometry and color, membership class, and effective temperature. In addition we include the orbital period and eccentricity for binary BSSs with orbital solutions.

The binary parameters of the SB1 systems are striking. All but one of the SB1 BSSs have periods within a factor of 2-3 of 1000 days, compared to the main sequence period distribution that extends from a few days up to several thousand days (Mathieu \& Geller 2009). The statistical secondary mass distribution has a sharp peak at $0.5 M_{\odot}$ that is distinct from the mass distribution expected for main sequence secondary stars at the $99 \%$ confidence level. This suggests the companions are CO WDs, whose masses are typically around $0.5 M_{\odot}$ (Geller \& Mathieu 2011). These binary parameters are consistent with the BSSs forming through Case C mass transfer (Chen \& Han 2008).

We set out to observationally detect these potential WD companions with a far-ultraviolet (FUV) photometric campaign using the Hubble Space Telescope $(H S T)$. The first results of this study are presented in Gosnell et al. (2014), where we identify three BSSs with hot WD companions and present specific formation scenarios for each system. The shorter period of WOCS 5379 of 120 days opens the possibility of Case B rather than Case C mass transfer, which is also explored in 
TABLE 1

NGC 188 Blue Straggler Star Population

\begin{tabular}{|c|c|c|c|c|c|c|c|c|c|}
\hline \multirow{2}{*}{\multicolumn{2}{|c|}{$\frac{\text { ID } \quad \text { Position }(\mathrm{J} 2000)}{\text { Non-velocity variable: }}$}} & $V^{\mathrm{a}}$ & $B-V^{\mathrm{a}}$ & Class $^{\mathrm{b}}$ & $T_{\mathrm{eff}}^{\mathrm{c}}$ & \multicolumn{2}{|c|}{$P_{\text {orb }}(\text { days })^{\mathrm{b}}$} & \multicolumn{2}{|c|}{ Eccentricity $^{\mathrm{b}}$} \\
\hline & & & & & & \multirow{2}{*}{\multicolumn{2}{|c|}{$\ldots$}} & \multirow{2}{*}{\multicolumn{2}{|c|}{$\ldots$}} \\
\hline 1366 & $00: 51: 15.06,+85: 44: 02.02$ & 15.851 & 0.620 & SM & $6120 \pm 120$ & & & & \\
\hline 4290 & $00: 42: 06.53,+85: 16: 47.25$ & 14.174 & 0.584 & SM & $6280 \pm 90$ & \multicolumn{2}{|r|}{$\ldots$} & \multicolumn{2}{|r|}{$\ldots$} \\
\hline 4306 & $00: 42: 20.59,+85: 15: 39.47$ & 13.347 & 0.534 & $\mathrm{SM}$ & $6450 \pm 100$ & \multicolumn{2}{|r|}{$\ldots$} & \multicolumn{2}{|r|}{$\ldots$} \\
\hline 5020 & $00: 47: 51.46,+85: 15: 09.09$ & 14.000 & 0.502 & $\mathrm{SM}$ & $6750 \pm 130$ & \multicolumn{2}{|r|}{$\ldots$} & \multirow{2}{*}{\multicolumn{2}{|c|}{$\ldots$}} \\
\hline \multicolumn{8}{|c|}{ Single-lined binaries: } & & \\
\hline 451 & $00: 34: 47.95,+85: 32: 27.33$ & 13.880 & 0.604 & BM & $6400 \pm 110$ & 722 & \pm & 0.34 & \pm 0.03 \\
\hline 1888 & $00: 54: 31.35,+85: 32: 09.12$ & 14.841 & 0.552 & $\mathrm{BM}$ & $6570 \pm 120$ & 2240 & \pm 30 & 0.21 & \pm 0.04 \\
\hline 2679 & $00: 26: 44.64,+85: 18: 35.94$ & 15.011 & 0.515 & $\mathrm{BM}$ & $6630 \pm 120$ & 1033 & \pm & \multicolumn{2}{|l|}{0.07} \\
\hline 4230 & $00: 43: 23.81,+85: 20: 32.64$ & 15.080 & 0.534 & $\mathrm{BU}$ & $6350 \pm 110$ & \multicolumn{2}{|r|}{$\ldots$} & \multirow{2}{*}{\multicolumn{2}{|c|}{$0.09 \stackrel{\cdots}{ \pm 0.05}$}} \\
\hline 4348 & $00: 43: 41.47,+85: 13: 17.28$ & 14.681 & 0.466 & $\mathrm{BM}$ & $6750 \pm 120$ & 1168 & \pm & & \\
\hline 4540 & $00: 45: 18.27,+85: 19: 19.85$ & 13.857 & 0.521 & BM & $6590 \pm 100$ & 3030 & \pm 70 & \multicolumn{2}{|c|}{$0.36 \pm 0.07$} \\
\hline 4581 & $00: 45: 56.63,+85: 17: 29.66$ & 14.147 & 0.536 & $\mathrm{BM}$ & $6750 \pm 100$ & 546.7 & 1.6 & \multicolumn{2}{|c|}{$0.269 \pm 0.015$} \\
\hline 4589 & $00: 46: 22.99,+85: 17: 13.46$ & 14.995 & 0.595 & $\mathrm{BM}$ & $6240 \pm 100$ & 615.2 & 1.7 & \multicolumn{2}{|c|}{$0.21 \pm 0.04$} \\
\hline 4970 & $00: 47: 13.32,+85: 16: 39.87$ & 14.524 & 0.622 & $\mathrm{BM}$ & $6170 \pm 90$ & 1002.76 & 2.4 & \multicolumn{2}{|c|}{$0.095 \pm 0.013$} \\
\hline 5325 & $00: 49: 36.86,+85: 16: 38.85$ & 14.978 & 0.605 & $\mathrm{BM}$ & $6060 \pm 100$ & 1772 & \pm 12 & \multicolumn{2}{|c|}{$0.77 \pm 0.03$} \\
\hline 5350 & $00: 49: 03.26,+85: 15: 25.19$ & 13.453 & 0.495 & $\mathrm{BM}$ & $6720 \pm 120$ & 690 & \pm & \multicolumn{2}{|c|}{$0.07 \pm 0.03$} \\
\hline 5379 & $00: 50: 10.79,+85: 14: 38.08$ & 15.372 & 0.570 & $\mathrm{BM}$ & $6400 \pm 120$ & 120.21 & 0.04 & \multicolumn{2}{|c|}{$0.24 \pm 0.03$} \\
\hline 5434 & $00: 48: 54.45,+85: 12: 36.71$ & 14.687 & 0.526 & $\mathrm{BM}$ & $6550 \pm 120$ & 1277 & \pm 9 & \multicolumn{2}{|c|}{$0.551 \pm 0.018$} \\
\hline 5671 & $00: 52: 25.78,+85: 15: 27.88$ & 13.600 & 0.659 & $\mathrm{BM}$ & $6130 \pm 90$ & 1423 & \pm 7 & \multicolumn{2}{|c|}{$0.286 \pm 0.018$} \\
\hline 8104 & $00: 40: 15.45,+85: 03: 48.49$ & 15.842 & 0.616 & $\mathrm{BM}$ & $6170 \pm 130$ & 2140 & \pm 110 & \multicolumn{2}{|c|}{$0.20 \pm 0.07$} \\
\hline \multicolumn{10}{|c|}{ Double-lined binaries: } \\
\hline 5078 & $00: 47: 11.69,+85: 13: 31.53$ & 14.465 & 0.601 & $\mathrm{BM}$ & $\ldots$ & \multirow{2}{*}{\multicolumn{2}{|c|}{$\begin{array}{l}4.78303 \pm 0.00012 \\
5.32770+0.00005\end{array}$}} & \multirow{2}{*}{\multicolumn{2}{|c|}{$\begin{array}{l}0.121 \pm 0.0006 \\
0.013 \pm 0.006\end{array}$}} \\
\hline 7782 & $00: 35: 46.23,+84: 57: 14.35$ & 14.379 & 0.486 & $\mathrm{BM}$ & $\ldots$ & & & & \\
\hline
\end{tabular}

${ }^{\text {a }}$ Sarajedini et al. (1999).

${ }^{\mathrm{b}}$ Geller et al. (2009). Classification classes are SM: Single Member, BM: Binary Member, and BU: Binary proper motion member, but no orbit or RV membership.

${ }^{\mathrm{C}}$ Calculated using 4- or 5-band color information following Ramírez \& Meléndez (2005), see Section 3.2

Gosnell et al. (2014).

In this paper we present the results of the HST study for the remaining BSS population in NGC 188 and discuss the implications for the frequency of different BSS formation mechanisms. We present our observations in Section 2, our photometric analysis of the BSS population and detection of WD companions in Section 3 discuss the frequency of mass-transfer formation and implications for modeling efforts in Section 4, and summarize our study in Section 5 .

\section{OBSERVATIONS}

Our observational design is described in Gosnell et al. (2014), but the salient details are repeated here for clarity. 19 of the $21 \mathrm{BSSs}$ in NGC 188 were observed using the Hubble Space Telescope Advanced Camera for Surveys (ACS) in the Solar Blind Channel (SBC). The observations occurred in 19 separate 2-orbit visits between 2012 October 3 and 2012 November 23 (GO: 12492, PI: Mathieu). We did not include the two SB2 BSSs in this study, as their high mass ratios and optical detection of the secondary stars indicate the companions are not WDs (Geller et al. 2009; Mathieu \& Geller 2009). An additional star, WOCS 1947, was included in the HST study but Mathieu \& Geller (2014) later found it to be a red giant star. A $V$-band photometric error in the literature caused the erroneous categorization of this source. We return to WOCS 1947 in Section 2.1.1.

Each BSS was observed in F140LP for 2040 s, F150LP for $2380 \mathrm{~s}$, and F165LP for $1564 \mathrm{~s}$. The total exposure times are the sum of four shorter exposures using the standard ACS/SBC 4-point box dither pattern. We also utilize derived narrow bandpasses to better isolate the bluest FUV flux. We exploit the nested nature of the long-pass filters by differencing F140LP and F150LP to create F140N, and differencing F150LP and F165LP to create F150N (Dieball et al. 2005; Gosnell et al. 2014).

The SBC field of view is very small at $25^{\prime \prime} \times 25^{\prime \prime}$. In all but one case the BSS target is the only star visible in the SBC image; therefore the images do not suffer from source confusion or blending.

\subsection{ACS/SBC Aperture Photometry}

Aperture photometry is carried out on redrizzled images with a pixel scale of $0 . \prime 025$ pixel $^{-1}$. We extract count rates using an aperture radius of 6 pixels, or 0 "' 15 , using the IRAF package DAOPHOT. We calculate encircled energy fractions for this aperture size using modeled Tiny Tim point source: 9 . We find corrected count rates by dividing the measured count rate by the encircled energy corrections of $0.83,0.84$, and 0.85 for F140LP, F150LP, and F165LP, respectively.

\subsubsection{ACS Red Leak}

The ACS/SBC has a known red leak beyond $2000 \AA$ (Boffi et al. 2008). Recent work has also shown that the red leak model included in SYNPHOT is no longer accurate (Feldman et al. 2010). Although the SBC detector temperature rises with continued use, we do not find any systematic correlation between flux and detector temperature across the four dithered exposures for each target.

\footnotetext{
${ }^{9}$ http://www.stsci.edu/hst/observatory/focus/TinyTim
} 
In the absence of identifying a red leak trend we use the serendipitous observation of the red giant WOCS 1947 to account for red leak contamination. As a red giant $(V=12.54, B-V=1.29$; Henden \& Munari 2014), we assume all of the SBC flux for WOCS 1947 is due to the red leak. We estimate the red leak contribution of each BSS by scaling the detected red leak flux of WOCS 1947 in each long-pass filter by $V$-band luminosity. We then subtract off the "extra" red leak flux. This results in count rates that, to the best of current capabilities, are red leak-free. The mean red leak correction in F150LP is $0.15 \mathrm{mag}$.

The detected red leak flux for WOCS 1947 is about twice the predicted flux from current SYNPHOT throughput curves. Modeling WOCS 1947 with an ATLAS9 spectrum (Castelli \& Kurucz 2004) with an effective temperature of $4575 \mathrm{~K}$ and surface gravity of $\log (g)=$ 1.5, the SYNPHOT task CALCPHOT calculates a count rate of 0.12 counts $\mathrm{s}^{-1}$ in all three long-pass filters. This is in comparison to the measured count rates of $0.212 \pm 0.009,0.279 \pm 0.009$, and $0.23 \pm 0.01$ counts $\mathrm{s}^{-1}$ for F140LP, F150LP, and F165LP, respectively. The resulting magnitude difference is 0.9 STMAG in F150LP $\left(M_{\text {SYNPHOT }}=22.1, M_{\mathrm{obs}}=21.2\right)$. These observations also suggest that the red leak is not constant across the long-pass filters. The measured difference of the observed red leak compared to SYNPHOT is slightly less than the difference seen by Feldman et al. (2010). Using the SBC PR130L prism, they find the observed red leak throughput to be 2.5 times that of the SYNPHOT throughput curve.

\subsubsection{Magnitude Calculation}

Encircled energy-corrected and red leak-subtracted count rates are used to calculate instrumental magnitudes in the STMAG system. We convert the count rates into fluxes $\left(\mathrm{erg} \mathrm{cm} \mathrm{cm}^{-2} \mathrm{~s}^{-1} \AA^{-1}\right.$ ) using the PHOTFLAM conversion factors for F140LP, F150LP, and F165LP provided in the ACS Data Handbook Gonzaga et al. 2013). We find the count rates in F140N and F150N by differencing the corrected count rates of the longpass filters, where $\mathrm{F} 140 \mathrm{~N}=(\mathrm{F} 140 \mathrm{LP}-\mathrm{F} 150 \mathrm{LP})$ and $\mathrm{F} 150 \mathrm{~N}=(\mathrm{F} 150 \mathrm{LP}-\mathrm{F} 165 \mathrm{LP})$. We calculate the flux conversion factors for F140N and F150N using SYNPHOT and the F140N and F150N flux errors by combining the flux errors of the long-pass filters in quadrature, scaled by the different exposure times. We calculate instrumental magnitudes in all bandpasses using STMAG $=-2.5 \log _{10}$ (flux) -21.1 . The photometric errors are dominated by Poisson noise due to the low background of the ACS/SBC MAMA detector, although the signal is high enough that the error distributions are approximately symmetric for all but the faintest measurements in F140N and F150N.

The FUV information for the 19 BSS in this study are presented in Table 2 with the WOCS ID, NUV magnitude, and 5-band FUV magnitudes (F140LP, F150LP, F165LP, F140N, and F150N). A F140N magnitude is only reported for those sources with a higher count rate in F140LP than F150LP. Magnitudes given in italics are less than $3 \sigma$ detections.

\subsection{WFC3 F218W Photometry}

Accurate modeling of the BSS population is a key factor in determining the amount of expected FUV emission. We take special care to match modeled and observed near-UV (NUV) photometry (see Section 3.2). We use GALEX NUV photometry for this purpose (Martin et al. 2005). However, one BSS in NGC 188, WOCS 8104, is not detected in GALEX. We obtained NUV photometry for WOCS 8104 using the HST Wide Field Camera 3 (WFC3) in F218W. The observation occurred on 2012 June 19 as part of this program, with a total exposure time of $669 \mathrm{~s}$. We use DAOPHOT for source detection and aperture photometry, and calculate the instrumental F218W magnitude for WOCS 8104 (given in Table 2).

\section{ANALYSIS}

\subsection{The FUV Color-Magnitude Diagram}

Identifying WD companions relies on detecting a FUV excess above the emission expected for a BSS alone. We first compare the observed photometry with modeled BSS photometry for the entire BSS population. This approach best demonstrates the general trend of FUV emission for the type of BSSs found in NGC 188 in comparison to the FUV emission for potential BSS+WD pairs. It also clearly reveals those BSSs with highly significant FUV excesses indicative of hot (temperatures greater than $12,000 \mathrm{~K}$ ) WD companions.

In Figure 1 we plot a FUV color-magnitude diagram (CMD) for the BSSs in our study along with photometric models of the total BSS population and BSS+WD pairings (described in detail in the next two sections). The observed BSS photometry is shown with black points and $1 \sigma$ error bars. Since we use F150N-F165LP as our color diagnostic we exclude any BSS with less than a $3 \sigma$ detection in F150N in Figure 1 and in all subsequent analyses. This removes WOCS 4589 and 5434, both binary stars, and WOCS 1366, a non-velocity variable star. The lack of a F150N detection in these three sources is consistent within $3 \sigma$ for each of the BSSs given their faint NUV magnitudes.

The remaining BSS sample used for further analysis includes 16 sources: 13 SB1 binaries and three nonvelocity variable stars. The only three sources also having more than a $3 \sigma$ detection in F140N (WOCS 4540 , 4348, and 5379) were presented in Gosnell et al. (2014), and are included in this sample of $16 \mathrm{BSSs}$.

\subsection{Modeling the Blue Straggler Star Population}

We calculate the FUV emission of the overall NGC 188 BSS population using a population synthesis approach including a large number of model spectra spanning the entire range of observed BSS temperatures and luminosities. The modeling is done through a three step process: (1) we establish the ranges of physical parameters that define the NGC 188 BSS population, (2) we create a large sample of model spectra constrained by those ranges, and (3) we calculate synthetic photometry for the sample of model spectra.

First, we define the range of parameters for the model spectra using the known temperatures and luminosities of the NGC 188 BSSs.

The BSS temperatures are determined using metallicity-dependent 4 - or 5 -band color-to- $T_{\text {eff }}$ 
TABLE 2

HST FUV Рнотомetry of NGC 188 BSS Population

\begin{tabular}{|c|c|c|c|c|c|c|}
\hline WOCS ID & $\mathrm{NUV}^{\mathrm{a}}$ & F165LP & F150LP & F140LP & $\mathrm{F} 150 \mathrm{~N}^{\mathrm{b}}$ & $\mathrm{F} 140 \mathrm{~N}^{\mathrm{b}}$ \\
\hline 451 & $18.38 \pm 0.04$ & $18.94 \pm 0.04$ & $20.06 \pm 0.03$ & $20.54 \pm 0.03$ & $22.2_{-0.8}^{+0.5}$ & $23.2_{-2.0}^{+0.7}$ \\
\hline 1366 & $20.6 \pm 0.2$ & $20.63 \pm 0.07$ & $21.83 \pm 0.05$ & $22.62 \pm 0.06$ & $25.5_{-1.7}^{+1.7}$ & $\ldots$ \\
\hline 1888 & $19.39 \pm 0.07$ & $19.78 \pm 0.05$ & $20.72 \pm 0.04$ & $21.37 \pm 0.04$ & $21.9_{-0.4}^{+0.3}$ & $\ldots$ \\
\hline 2679 & $19.07 \pm 0.06$ & $19.22 \pm 0.04$ & $20.21 \pm 0.03$ & $20.67 \pm 0.03$ & $21.5_{-0.3}^{+0.3}$ & $22.7_{-0.8}^{+0.4}$ \\
\hline 4230 & $19.49 \pm 0.07$ & $19.38 \pm 0.04$ & $20.33 \pm 0.03$ & $20.77 \pm 0.03$ & $21.5_{-0.3}^{+0.2}$ & $22.6_{-0.6}^{+0.4}$ \\
\hline 4290 & $19.02 \pm 0.06$ & $19.23 \pm 0.04$ & $20.32 \pm 0.03$ & $20.90 \pm 0.03$ & $22.3_{-0.7}^{+0.4}$ & $\ldots$ \\
\hline 4306 & $18.00 \pm 0.04$ & $18.23 \pm 0.03$ & $19.29 \pm 0.02$ & $19.81 \pm 0.02$ & $20.9_{-0.3}^{+0.2}$ & $\ldots$ \\
\hline 4348 & $18.59 \pm 0.05$ & $18.64 \pm 0.03$ & $19.55 \pm 0.02$ & $19.96 \pm 0.02$ & $20.6_{-0.2}^{+0.2}$ & $21.4_{-0.3}^{+0.2}$ \\
\hline 4540 & $18.17 \pm 0.04$ & $17.96 \pm 0.02$ & $18.58 \pm 0.01$ & $18.80 \pm 0.01$ & $19.07_{-0.07}^{+0.06}$ & $19.29_{-0.05}^{+0.05}$ \\
\hline 4581 & $18.47 \pm 0.04$ & $18.74 \pm 0.03$ & $19.82 \pm 0.02$ & $20.41 \pm 0.03$ & $21.6_{-0.5}^{+0.3}$ & $\ldots$ \\
\hline 4589 & $19.77 \pm 0.09$ & $20.18 \pm 0.06$ & $21.34 \pm 0.05$ & $21.86 \pm 0.05$ & $24.1_{-1.0}^{+1.0}$ & $\ldots$ \\
\hline 4970 & $19.63 \pm 0.08$ & $19.95 \pm 0.06$ & $20.96 \pm 0.04$ & $21.53 \pm 0.05$ & $22.4_{-0.5}^{+0.4}$ & $\ldots$ \\
\hline 5020 & $18.09 \pm 0.04$ & $18.19 \pm 0.03$ & $19.21 \pm 0.02$ & $19.71 \pm 0.02$ & $20.7_{-0.2}^{+0.2}$ & $22.7_{-1.6}^{+0.6}$ \\
\hline 5325 & $19.78 \pm 0.09$ & $19.96 \pm 0.05$ & $20.98 \pm 0.04$ & $21.63 \pm 0.04$ & $22.5_{-0.5}^{+0.4}$ & $\ldots$ \\
\hline 5350 & $17.63 \pm 0.03$ & $17.50 \pm 0.02$ & $18.49 \pm 0.01$ & $18.97 \pm 0.01$ & $19.9_{-0.1}^{+0.1}$ & $21.4_{-0.4}^{+0.3}$ \\
\hline 5379 & $19.87 \pm 0.09$ & $19.13 \pm 0.04$ & $19.23 \pm 0.02$ & $19.26 \pm 0.02$ & $19.27_{-0.06}^{+0.05}$ & $19.31_{-0.04}^{+0.04}$ \\
\hline 5434 & $19.00 \pm 0.06$ & $19.36 \pm 0.04$ & $20.53 \pm 0.03$ & $21.13 \pm 0.04$ & $23.4-0.9$ & $\cdots$ \\
\hline 5671 & $18.80 \pm 0.05$ & $19.09 \pm 0.04$ & $20.17 \pm 0.02$ & $20.73 \pm 0.03$ & $22.0_{-0.6}^{+0.4}$ & $\ldots$ \\
\hline 8104 & $19.69 \pm 0.02^{\mathrm{c}}$ & $21.27 \pm 0.11$ & $22.15 \pm 0.07$ & $23.01 \pm 0.09$ & $23.1_{-0.6}^{+0.4}$ & $\ldots$ \\
\hline
\end{tabular}

${ }^{\mathrm{a}}$ GALEX NUV magnitude (AB system), except where noted (Martin et al. 2005).

${ }^{\mathrm{b}}$ Magnitudes in italic text are less than $3 \sigma$ detections.

${ }^{\mathrm{c}}$ WFC3 F218W instrumental magnitude (this study).

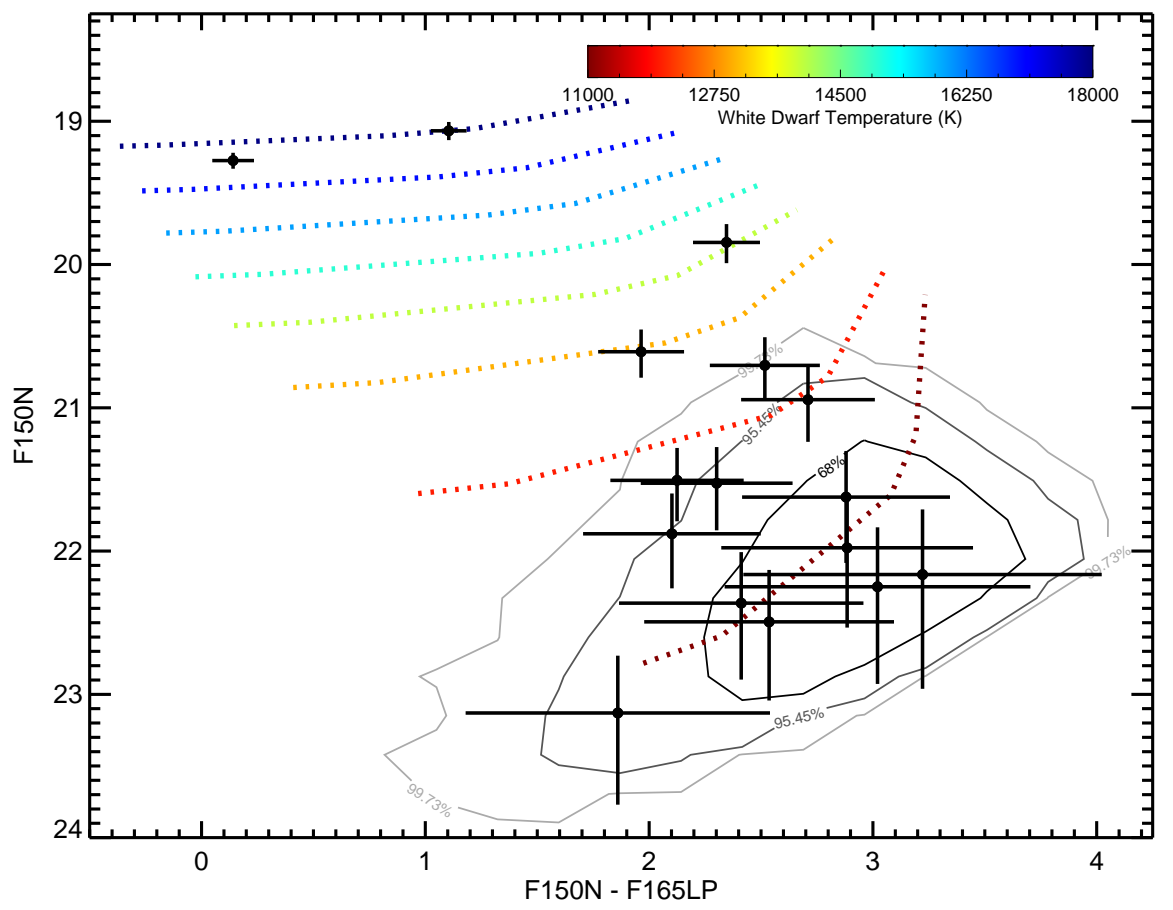

FIG. 1. - FUV CMD of the NGC 188 BSSs, shown in black with $1 \sigma$ error bars. The three BSSs with less than a $3 \sigma$ detection in F150N are not included in this figure. The BSS-only model distribution is shown with grey contours at the 1, 2, and $3 \sigma$ level, as labeled in the figure. The rainbow-colored tracks show synthetic photometry of representative BSS+WD spectra with dotted lines showing tracks of constant WD temperature. The WD temperatures range from 11,000 to 18,000 K, as indicated by the color bar, with BSS temperatures varying from $5750 \mathrm{~K}$ (red end) to $6750 \mathrm{~K}$ (blue end) along each dashed line. Four BSSs are clearly seen to have a significant FUV excess and are separated from the BSS-only distribution by more than $3 \sigma$ (see also Gosnell et al. 2014). 
conversions from Ramírez \& Meléndez (2005). The colors used are $B-V, V-J, V-H$, and $V-K$ (Sarajedini et al. 1999; Skrutskie et al. 2006). $V-I$ is also used when $I$ photometry is available. The resulting temperatures and errors for the non-velocity variable and SB1 BSSs are given in Table 1. The errors given include the error in each color measurement and the systematic error in the temperature calculation as given in Ramírez \& Meléndez (2005).

We set the luminosity range based on GALEX NUV magnitudes for the BSSs, which vary from $20.6 M_{\mathrm{NUV}} \mathrm{AB}$ to $18.0 M_{\mathrm{NUV}, \mathrm{AB}}$ (Martin et al. 2005). As GALEX NUV photometry is the bluest luminosity information available it provides the most accurate normalization for estimating the FUV flux.

Next, we create 50,000 individual model spectra that, together, constitute the BSS-only model population. We create smooth probability density functions (PDFs) modeled after the observed shapes of BSS temperature and NUV luminosity cumulative distribution functions (CDFs). We Monte Carlo sample the PDFs to determine the parameters used for each model spectrum. There is only a weak correlation between temperature and luminosity in the NGC 188 BSS population $(r=$ -0.2 ), so the two parameters are sampled independently. For each sampling of the PDFs we calculate a model spectrum by interpolating between reddened UVBLUE spectra with temperatures of 6000, 6500, 6750, and $7000 \mathrm{~K}$ to match the sampled temperature $(\mathrm{E}(B-V)$ $=0.09,[\mathrm{Fe} / \mathrm{H}]=0, \log (g)=3.5 ;$ Sarajedini et al. 1999; Rodríguez-Merino et al. 2005). We then normalize the interpolated spectrum to match the sampled NUV luminosity. The interpolation and normalization steps are carried out on all 50,000 PDF samples. As a result, we have a BSS-only model composed of a large set of model spectra spanning the range of physical parameters observed in the NGC 188 population.

Finally, after creating the BSS-only model we calculate the expected FUV CMD for the entire model distribution. We convolve each model spectrum with SYNPHOT F140LP, F150LP, and F165LP throughput curves to obtain count rates. Since the observed data are red leakcorrected the throughput curves do not include a red leak component. We add Poisson noise to the convolved count rates to mimic photometric errors. The count rates and flux errors in F140N and F150N are calculated in the same manner as the observations. We calculate magnitudes in F140LP, F150LP, F165LP, F140N, and F150N for each of the 50,000 modeled spectra.

We compare the modeled BSS-only FUV photometry with the observed BSSs in Figure 1. The 2-dimensional density histogram of modeled photometry is represented by the gray contours encompassing 68\%, 95.45\%, and $99.73 \%$ of the distribution, as labeled. We restrict the density contours to those modeled sources that meet or exceed the same $3 \sigma \mathrm{F} 150 \mathrm{~N}$ detection threshold we apply to the observations 10 .

10 We note that a similar figure in Gosnell et al. (2014) does not have the same $3 \sigma$ detection cutoff, resulting in contours that extend fainter and redder than the contours shown here. In either case, the extent of the contours into the lower right corner of the CMD does not impact our search for WD companions.

\subsection{Sources with Significant FUV Excesses}

In order to demonstrate how the presence of a WD companion changes the expected FUV emission we create representative pairs of $\mathrm{BSS}+\mathrm{WD}$ binaries. Keeping the BSS luminosity constant at a GALEX NUV magnitude of 18.0 to mimic the brightest BSS in the NUV, we add together the spectra of BSSs between $5750 \mathrm{~K}$ and $6750 \mathrm{~K}$ (Rodríguez-Merino et al. 2005) with WDs of increasing temperature, ranging from $11,000 \mathrm{~K}$ to $18,000 \mathrm{~K}$. We use WD spectra with a $\log (g)=7.75$ (P. Bergeron, private communication; Wood 1995). We calculate the synthetic photometry for each BSS+WD pair using the same method as for the BSS-only distribution.

The synthetic photometric results for the BSS+WD pairs are shown in Figure 1 as dotted lines. The rainbow colors indicate the WD temperature, as shown with the color bar. The length of each line shows the photometry of that particular WD temperature with a BSS of 5750 $\mathrm{K}$ on the left (blue end) extending to a BSS of 6750 $\mathrm{K}$ on the right (red end). Since a higher temperature BSS contributes more light in F165LP it results in an overall redder color. The position of the rainbow tracks changes slightly with the choice of BSS luminosity, but a single BSS luminosity is sufficient to illustrate the general photometric trends for BSS+WD binaries.

Visual analysis of Figure 1 shows there are four sources with obvious FUV excesses: WOCS 4348, WOCS 4540, WOCS 5350, and WOCS 5379. These sources are well separated from the BSS-only distribution and their photometry is consistent with the FUV excesses expected for BSS+WD binaries.

We fit BSS+WD spectra to each of the four FUV excess sources individually to estimate the companion WD temperature. In Figure 2 we show the F140N, F150N, F165LP, and GALEX NUV photometric data for these sources along with the best-fit BSS+WD spectrum found through weighted least squares minimization. A similar figure for WOCS 4348, 4540, and 5379 is shown in Gosnell et al. (2014), although here we employ an improved method of finding the best-fit WD temperature. The grid of WD models (P. Bergeron, private communication) has a temperature resolution of $1,000 \mathrm{~K}$. In Gosnell et al. (2014) we restricted our search to grid temperature values while in this work we interpolate between temperatures. Given the uncertainty in the best-fit WD temperatures, the results here and in Gosnell et al. (2014) are consistent. In each case the mean temperature BSS spectrum is shown in orange, the WD spectrum in blue, and the combined spectrum in gray. The synthetic photometry of the combined spectrum is shown with the light gray boxes. The horizontal extent of the boxes shows the filter width while the vertical extent shows the range in photometry, including the BSS and WD temperature uncertainties. The combined spectrum is required to fit the GALEX NUV photometry. The narrow-band effective throughputs are shown in the upper left plot as a guide. The observed photometry is shown in black circles at the reference wavelength for each bandpass, and observed $3 \sigma$ photometric errors are shown when they exceed the symbol size. Measurements in F140N that are less than $3 \sigma$ are shown with an open circle. The best-fit spectra are also checked for consistency with $U$-band photometry, when available 

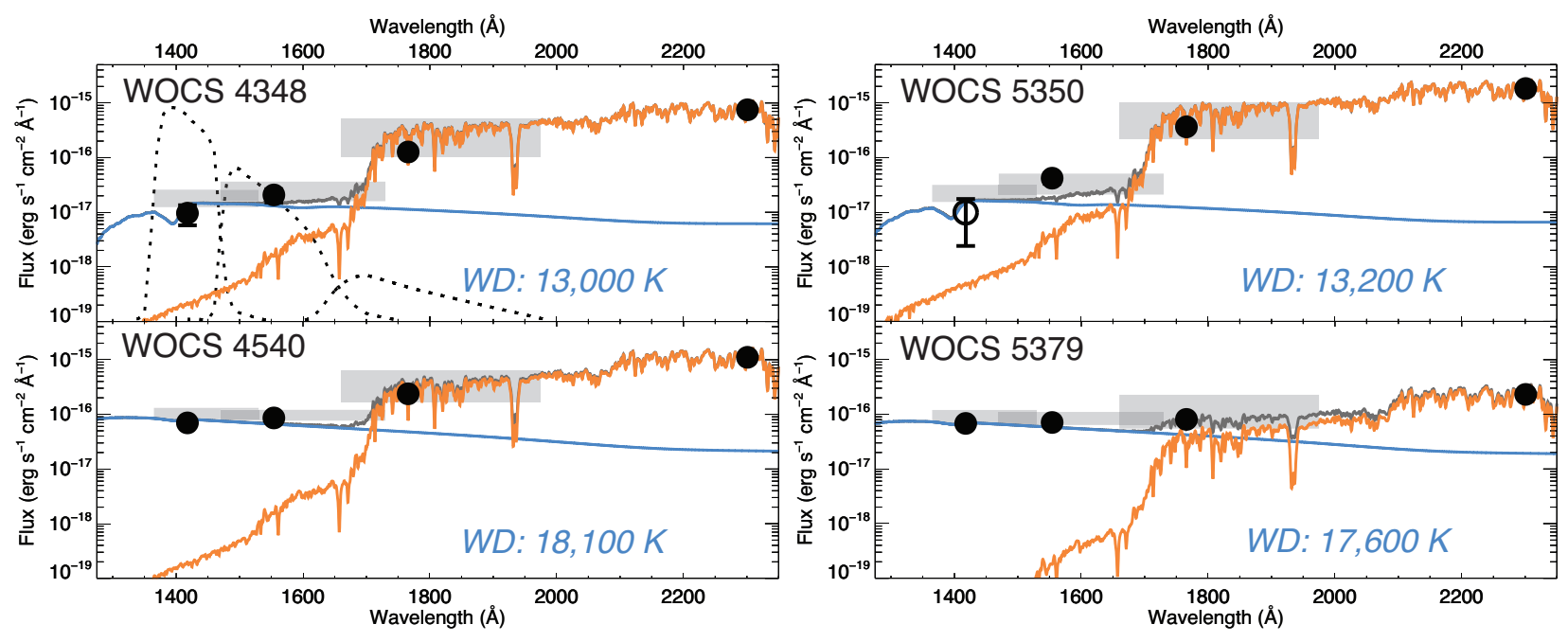

FIG. 2.- Best-fit BSS+WD spectra for the four BSS with hot WD companions, as labeled. The WD spectrum is shown in blue with the temperature as labeled, the mean BSS temperature spectrum (given in Table11) is shown in orange, and the combined BSS+WD spectrum is shown in dark gray. The synthetic photometry of the combined spectrum is shown with the light gray boxes. The vertical extent of the boxes shows the photometry range given the temperature uncertainty of the BSS and WD. The horizontal extent of the boxes represents the filter width, including the red tail of each bandpass. The upper left plot also includes the effective narrow-band throughputs (black dashed lines) as a guide. The observed photometry in F140N, F150N, F165LP, and GALEX NUV is shown as filled black circles at the reference wavelength for each bandpass. Measurements in F140N that are below $3 \sigma$ are shown as an open circle. Observed flux error bars $(3 \sigma)$ are plotted in black when the error exceeds the size of the symbol. The best-fit spectrum is required to match the GALEX NUV measurement.

(Sarajedini et al. 1999).

We use the estimated temperature and uncertainty from Figure 2 to find the range of WD ages for each source (Holberg \& Bergeron 2006; Tremblay et al.|2011). In Table 3 we list the best-fit WD temperature estimates and the age range for each of the four BSSs with a hot WD companion, along with the observed binary period and eccentricity (Geller et al. 2009). Of these BSSs, the maximum age is $230 \pm 30$ Myr for WOCS 4348, so all four BSSs formed very recently in comparison to the 7 Gyr age of NGC 188.

\subsection{The FUV-Faint BSS Sample}

There are 12 remaining BSS (nine binaries, three nonvelocity variables) within the sample of F150N sources that lack a significant (greater than $3 \sigma$ ) FUV excess. The possibility remains that some of these BSSs have a cool WD companion between 11,000-12,000 K. At these temperatures the WD emission would shift the observed photometry to be slightly bluer and brighter than the expected emission for that particular BSS. We cannot detect any WDs with temperatures below 11,000 K. Such "cold" WDs would not have enough FUV emission on their own to exceed the expected emission for any BSS in NGC 188.

We investigate whether any of the 12 FUV-faint BSSs contain a cool (11,000-12,000 K) WD companion by modeling the expected photometry for each BSS individually. Following a similar methodology as in Section 3.2 for each individual FUV-faint BSS we do a Monte Carlo sampling of the temperature range given in Table 1 and luminosity range, as constrained by GALEX NUV photometry for that BSS. The resulting sample is synthetically observed to create a 2-dimensional density distribution of expected FUV emission in F150N and F150N-F165LP for that particular BSS. We then find the model density value with the same color and magni- tude as the BSS observation, and adopt that value as the probability the BSS is consistent with the expected FUV emission of a BSS without a WD companion $\left(P_{\mathrm{BSS}}\right)$. The probability that the BSS has a WD companion is then $P_{\mathrm{WD}}=1-P_{\mathrm{BSS}}$.

The modeled photometry for each BSS is highly dependent on BSS temperature, which is more uncertain than the NUV luminosity used to normalize the model spectra. We compared the temperatures used here with recently calculated temperatures found from $\mathrm{H} \alpha$ lineprofile fits (K. Milliman, private communication). Many BSS temperatures are consistent across the methods, but WOCS 4230 and 4581 have temperature differences of approximately $200 \mathrm{~K}$. Additionally, the temperatures of the non-velocity variable BSSs WOCS 4306 and 5020 vary by $200-300 \mathrm{~K}$. If the actual BSS temperature is different from the modeled BSS temperature the observed BSS photometry could mimic the presence of a cool WD companion. In order to account for potential offsets in the known temperatures, and avoid false identification of a WD companion, we also model each BSS with temperature ranges shifted by $\pm 200 \mathrm{~K}$ and adopt the highest $P_{\mathrm{BSS}}$, or lowest $P_{\mathrm{WD}}$, for each BSS.

In Table 4 we list each FUV-faint BSS along with the probability that the source has a cool WD companion between 11,000-12,000 K. There are three BSS with probabilities greater than $2 \sigma$ : WOCS $1888\left(P_{\mathrm{WD}}=\right.$ $96 \%)$, WOCS $2679\left(P_{\mathrm{WD}}=97 \%\right)$, and WOCS 4230 $\left(P_{\mathrm{WD}}=99 \%\right)$. The photometry for WOCS 1888, 2679, and 4230 are best fit with BSS+WD composite spectra with WD temperatures between 11-12,000 K, as shown in Figure 3. The best-fit temperatures and corresponding ages for these sources, along with binary orbit parameters, are given in Table 5. There is a $2 \%$ chance that one of the $12 \mathrm{FUV}$-faint BSSs has a probability equal to or greater than $96 \%$ due to random fluctuations alone, but only a $0.03 \%$ chance that all three are due 
TABLE 3

Hot WD COMPANion TEMPERATURE AND AGE ESTIMATES

\begin{tabular}{cccccc} 
WOCS ID & WD temp $(\mathrm{K})$ & Age $(\mathrm{Myr})^{\mathrm{a}}$ & Period (days) $^{\mathrm{b}}$ & eccentricity $^{\mathrm{b}}$ \\
\hline 4348 & $13000 \pm 500$ & $230 \pm 30$ & 1168 & $\pm 0.09 \pm 0.05$ \\
4540 & $18100 \pm 500$ & $70 \pm 7$ & 3030 & \pm 70 & $0.36 \pm 0.07$ \\
5350 & $13200 \pm 500$ & $220 \pm 30$ & $690 \pm 3$ & $0.07 \pm 0.05$ \\
5379 & $17600 \pm 500$ & $77 \pm 7$ & $120.21 \pm 0.04$ & $0.24 \pm 0.03$ \\
\hline
\end{tabular}

${ }^{a}$ Holberg \& Bergeron (2006); Tremblay et al. (2011).

${ }^{\mathrm{b}}$ Geller et al. (2009).

TABLE 4

FUV-FAINT BSSs: Probability OF A COOL WD COMPANION

\begin{tabular}{|c|c|c|c|c|c|c|c|c|c|c|c|c|}
\hline WOCS ID : & 451 & 1888 & 2679 & 4230 & 4290 & 4306 & 4581 & 4970 & 5020 & 5325 & 5671 & 8104 \\
\hline$P_{\mathrm{WD}}:$ & 0.13 & 0.96 & 0.97 & 0.99 & 0.63 & 0.74 & 0.56 & 0.79 & 0.92 & 0.81 & 0.70 & 0.10 \\
\hline
\end{tabular}

to random fluctuations. We assume that these three BSSs have WD companions and formed through mass transfer between 310-360 Myr ago (Holberg \& Bergeron 2006; Tremblay et al. 2011).

For the remaining FUV-faint BSSs, the fit between the observed and modeled photometry is not improved by adding a cool WD of any temperature greater than $11,000 \mathrm{~K}$. The photometry of these sources is consistent with a BSS-only synthetic spectrum, and these sources do not have WD detections. The best fit spectra for the nine remaining BSSs with the corresponding $H S T$ and GALEX photometric points are shown in Figure 4

\section{THE NGC 188 BSS POPULATION}

The HST photometry reveals four hot WD companions hotter than $12,000 \mathrm{~K}$ and three additional WD companions between 11,000-12,000 K among 15 BSS SB1 binaries and four non-velocity variable BSSs. All of the detected WDs are in known binary systems. These data do not preclude the presence of cold WD companions (temperatures less than 11,000 K) among the remaining BSS, but we are unable to identify cold WD companions using these photometric data.

In Figure 5 we plot an optical CMD $(V, B-V)$ of NGC 188 member stars (Geller et al. 2008). The cluster zero-age main sequence (ZAMS) is shown as a solid black line. The BSSs are highlighted according to their binarity and WD companion temperature, which is a proxy for BSS age. Binary BSSs are plotted as diamonds and nonvelocity variable BSSs are plotted as large circles. The two SB2 BSSs, not part of this HST study, are shown as outlined black diamonds. The BSS binaries with WD detections are shown with a color from dark blue to light blue representing the measured WD temperature, as indicated with the color bar. The three BSSs with less than a $3 \sigma$ detection in F150N are shown outlined in light grey instead of black.

Since WD temperature correlates with age, we can use the CMD location of BSSs with detected WD companions to probe relationships between BSS age and optical luminosity and color. Several of the youngest BSSs (dark blue diamonds) sit along the ZAMS. BSS binaries along the ZAMS include two of the BSSs with hot WD companions (WOCS 4348 and 5379), two of the BSSs with cool WD companions (WOCS 2679 and
4230), and an additional binary with a $10 \%$ probability of having a WD companion (WOCS 8104). Based on the detected WD temperatures, the spread of ages along the ZAMS is 77-360 Myr (Holberg \& Bergeron 2006; Tremblay et al. 2011), and may extend further if WOCS 8104 has a cold and old WD companion.

At the same time, two of the youngest mass transferformed BSSs (WOCS 4540 and 5350) lie well off the ZAMS.

These results suggest that BSS products along the ZAMS may in fact be among the youngest in the population, but distance from the $Z A M S$ is not necessarily equivalent with age since formation. One must use caution when inferring the age of BSSs using single-star isochrones, especially for BSSs that lie away from the ZAMS.

Three of the four non-velocity variable BSSs are also among the most optically luminous of the population. If these BSSs formed through collisions they would likely be among the most massive BSSs, perhaps accounting for their high optical luminosity (Leigh \& Sills 2011). And yet, they are not more luminous than the most luminous mass transfer-formed BSSs. There is not a clear color or luminosity separation between mass transfer BSSs and possible collision BSSs in the optical CMD.

\subsection{Frequency of the Mass Transfer Formation Channel}

Previous work predicted that the majority of the NGC 188 binary BSSs formed through mass transfer (Mathieu \& Geller 2009; Geller \& Mathieu 2011). The seven WDs detected in this work indicate that more than half of the SB1 BSSs formed through mass transfer. Older mass transfer-formed BSSs have cold WD companions that cannot be detected with this photometric method, so the total number of mass transfer-formed BSSs is greater than seven. Therefore, our results confirm previous predictions that the majority of binary BSSs in NGC 188 formed through mass transfer.

We can further constrain the total frequency of masstransfer formation by considering how many BSSs may form through other formation mechanisms.

We do not expect any of the non-velocity variable BSSs to form through Case $\mathrm{B}$ or $\mathrm{C}$ Roche lobe overflow mass transfer. As non-velocity variable objects, these BSSs are either single stars or binary stars with extremely long 
TABLE 5

Cool WD COMPanion temperature AND AGe estimates

\begin{tabular}{|c|c|c|c|c|}
\hline WOCS ID & WD temp (K) & Age $(\mathrm{Myr})^{\mathrm{a}}$ & Period (days) ${ }^{\mathrm{b}}$ & eccentricity ${ }^{\mathrm{b}}$ \\
\hline 1888 & $11200 \pm 500$ & $360 \pm 50$ & $2240 \pm 30$ & $0.21 \pm 0.04$ \\
\hline 2679 & $11300 \pm 500$ & $350 \pm 50$ & $1033 \pm 8$ & $0.07 \pm 0.05$ \\
\hline $4230^{\mathrm{c}}$ & $11800 \pm 500$ & $310 \pm 40$ & $\ldots$ & $\ldots$ \\
\hline
\end{tabular}

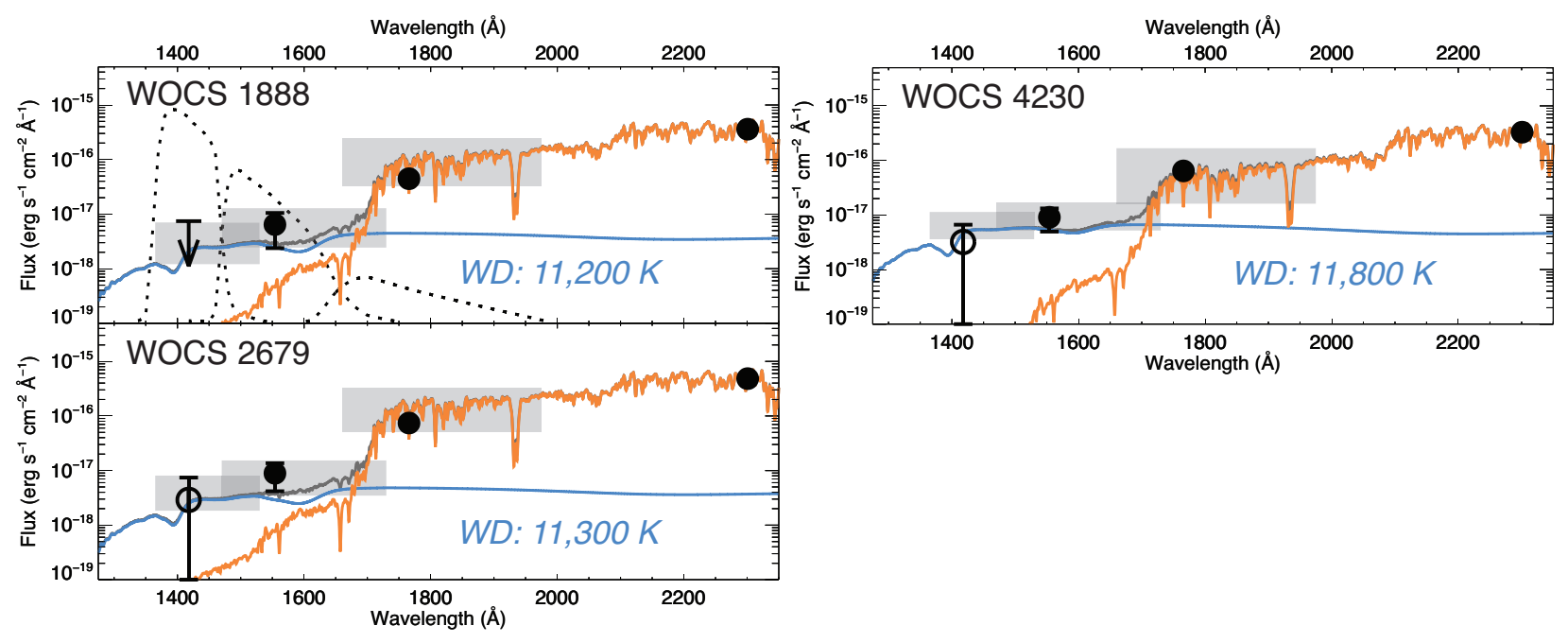

FIG. 3.- Best-fit BSS+WD spectra for the three BSS with cool (11,000-12,000 K) WD companions, as labeled. The colors and symbols used are the same as in Figure 2 For sources without a F140N detection the $3 \sigma$ upper limit is shown with a black bar and a down arrow. Due to the fainter WD flux these three BSSs have WD companions detected at the $2 \sigma$ level. The WD temperatures here correspond to ages between 310-360 Myr (Holberg \& Bergeron 2006; Tremblav et al. 2011).

periods greater than 10,000 days. Case C mass transfer systems have final orbital periods less than about 3,000 days (Chen \& Han 2008), which is well within the RV binary detection limit in NGC 188 (Geller et al. 2009). (We note that there is a small possibility for a nonvelocity variable source to be in a face-on binary that is undetectable using radial velocity methods. Incompleteness studies indicate there is a $24 \%$ chance that one of the non-velocity variable BSSs in NGC 188 is in a binary with a period less than $10^{4}$ days but is not detected due to inclination; Geller \& Mathieu 2012.) We propose that the non-velocity variable BSSs formed through other mechanisms, such as collisions or mergers from contact binaries.

Is it possible for any of the remaining eight SB1 BSS binaries to form through non-mass transfer processes? It is unlikely that the SB1 BSSs formed through collisions. BSSs formed through collisions can be in binaries, but the orbital periods tend to be very long and beyond RV sensitivity limits (Geller et al. 2013). In addition, given the observed statistical secondary mass distribution the SB1 BSS secondary masses are between $0.2-0.8 M_{\odot}$ (Geller \& Mathieu 2011), with a strong peak at $0.5 \mathrm{M}_{\odot}$. Models of collisionally-formed BSS binaries result in more massive companions than the observed secondary mass distribution allows (Geller \& Mathieu 2011).

Some SB1 BSSs may have formed through
Kozai-induced mergers in a hierarchical triple (Perets \& Fabrycky 2009; Naoz \& Fabrycky 2014). The resulting period distribution would be similar to that observed in the NGC 188 BSS binaries, but the secondary masses would differ (Perets 2014). The original tertiary, now the BSS secondary, would be drawn from the cluster initial-mass function (Kroupa 2001).

Without strong observational constraints on the primordial triple population in clusters it is difficult to make confident predictions about the number of Kozai-formed BSSs. Nonetheless, Geller et al. (2013) put limits on Kozai-BSS formation with an $N$-body model of NGC 188 containing 200 primordial triples — essentially giving every primordial binary with periods between 2 and 50 days a tertiary companion. This model creates an additional 1-2 BSSs at the age of NGC 188, but most of the BSSs result from collisions involving triple systems, not from the Kozai mechanism itself. Approximately 0.5 BSSs per model are created through the Kozai mechanism. However, the Geller et al. (2013) Kozai model prescriptions are possibly inaccurate such that the Kozai origin could be more prevalent than current $N$ body models suggest. We estimate that one of the BSS SB1 binaries may have formed through a merger in a hierarchical triple system.

We currently have no specific hypothesis for the for- 


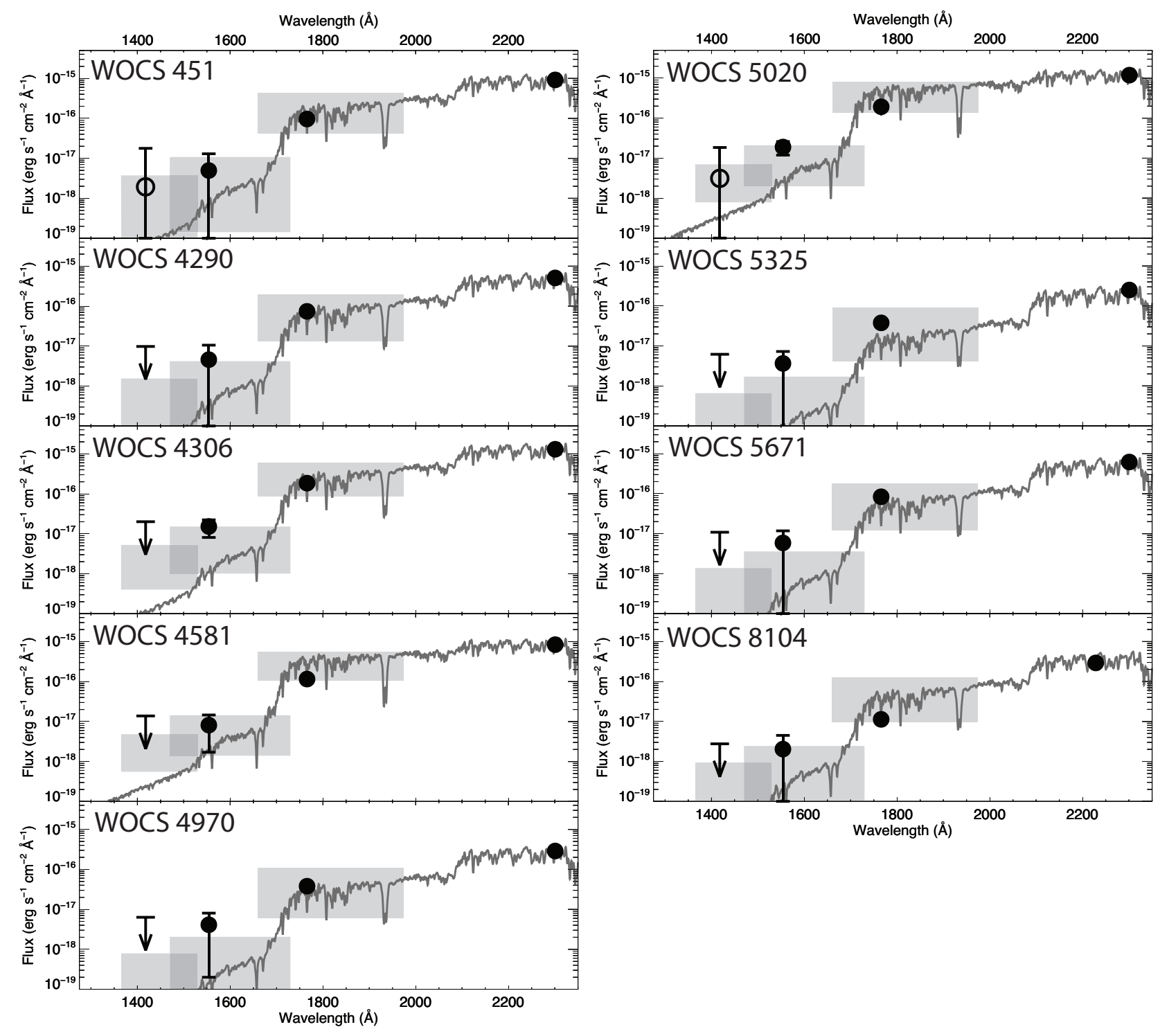

Fig. 4. - Best-fit spectra for the BSSs without significant FUV excesses. The colors and symbols used are the same as in Figures 2 and 3 These sources do not have detected WD companions with temperatures of 11,000 K or greater. (The NUV point for WOCS 8104 is WFC3 F218W photometry from this study.)

mation mechanism of the two SB2 short-period BSS binaries. Case $\mathrm{B}$ and $\mathrm{C}$ mass transfer cannot result in a short-period BSS binary with a mass ratio near unity, as seen in the case of WOCS 5078 and $7782 . \mathrm{N}$ body models fail to create similar systems, even with populations of collisionally formed BSSs (Hurley et al. 2005; Geller et al. 2013). These BSSs may have formed through any of the potential formation pathways and exchanged into their current binaries, but there is no way to know for certain. As they have more complex origins we exclude them from the following discussion of broad BSS population characteristics.

In summary, we detect WD companions to 7 of the 15 SB1 BSSs, establishing a lower limit of the mass-transfer formation frequency of $33 \%$. Given the binary properties and inferred companion masses of the remaining SB1 BSSs in NGC 188, our investigation of the currently hypothesized formation mechanisms suggests that 14 SB1 BSSs were formed through mass transfer. Thus, 14 of the 21 BSSs likely formed through mass transfer, for a total BSS mass-transfer formation frequency in NGC 188 of approximately $67 \%$.

\subsection{Consistency with Binary Population Synthesis Models}

Now that other BSS formation scenarios have been considered we can make an appropriate comparison between our empirical results and the theoretical results of the Geller et al. (2013) N-body model of NGC 188. Geller et al. (2013) present 20 full-lifetime $N$ body realizations of NGC 188, with complete tracking of the binary populations and dynamical encounters. The models create mass transfer-formed BSSs, and retain information on when the mass transfer ended in each case.

With seven WD detections we can directly compare the empirical and theoretical age distributions for mass transfer-formed BSSs. We create the $N$-body age dis- 


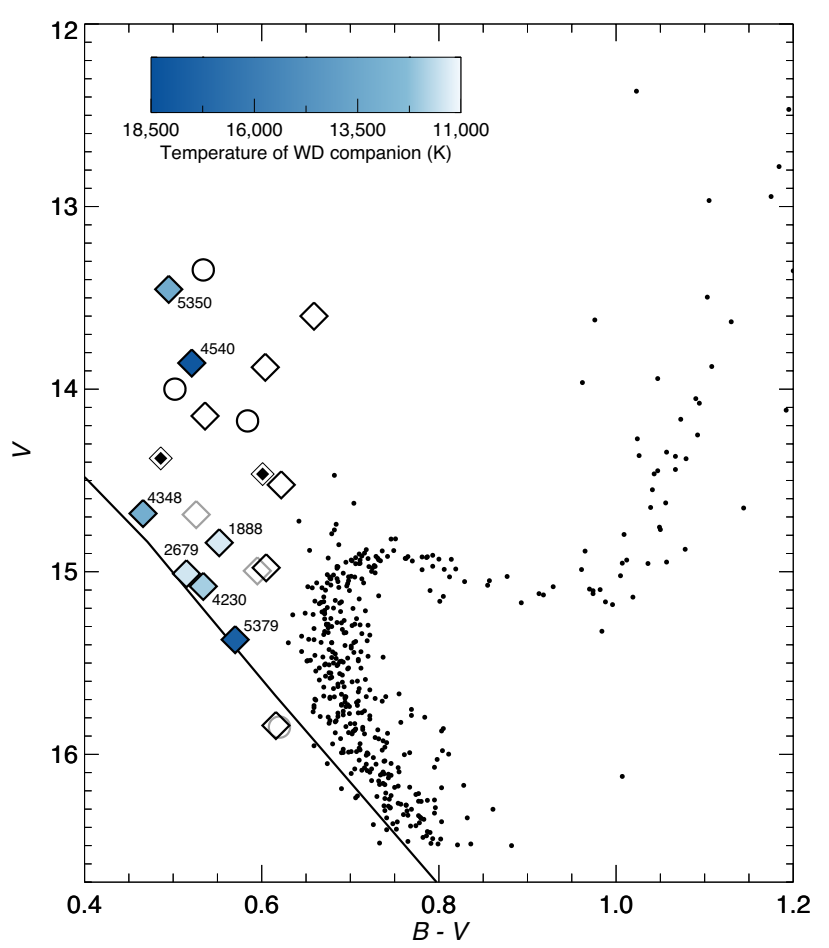

FIG. 5.- Optical CMD of NGC 188 cluster members with the BSS population highlighted according to binarity and the measured temperature of WD companions. The solid black line is the ZAMS for NGC 188. Binary BSSs are shown as diamonds and nonvelocity variable BSSs are shown as large circles. The outlined solid black diamonds are the two double-lined BSS binaries that are not included in this HST study. The BSSs with WD detections are shown with a color from dark blue to light blue representing the temperature of the WD companion, as indicated with the color bar. The sources outlined in grey (two SB1 binaries, one single BSS) are the three sources without a $3 \sigma$ detection in F150N.

tribution using all mass transfer-formed BSSs observable between 6.0-7.5 Gyr (Geller et al. 2013), and convert the time since mass transfer ended to a WD temperature (Holberg \& Bergeron 2006; Tremblay et al. 2011). The resulting CDF is shown in Figure 6 as a solid black line. The light gray lines are the result of Monte Carlo bootstrap resampling of the $N$-body CDF, meant to illustrate the inherent error in the $N$-body age distribution.

We compare the $N$-body age distribution with the empirical age distribution, shown in Figure [6 as blue squares with $500 \mathrm{~K}$ error bars. A KS test shows the empirical distribution and theoretical distribution are consistent with being drawn from the same parent distribution $(p=0.97)$. The overall shape of the $N$ body age distribution is fully consistent with the BSS ages detected in this study.

Adopting a mass-transfer formation frequency of $67 \%$ for the BSS population implies there are five BSSs in NGC 188 that formed through other formation mechanisms, such as collisions or the Kozai mechanism, in addition to the two short-period SB2 systems. This number is consistent with the number of collisionally formed BSSs seen in the Geller et al. (2013) $N$-body model of NGC 188.

The fraction of collisionally formed BSSs in the Geller et al. (2013) study is quite different than observed, however, because the total number of BSSs created is too low. The model results have an average of six BSSs

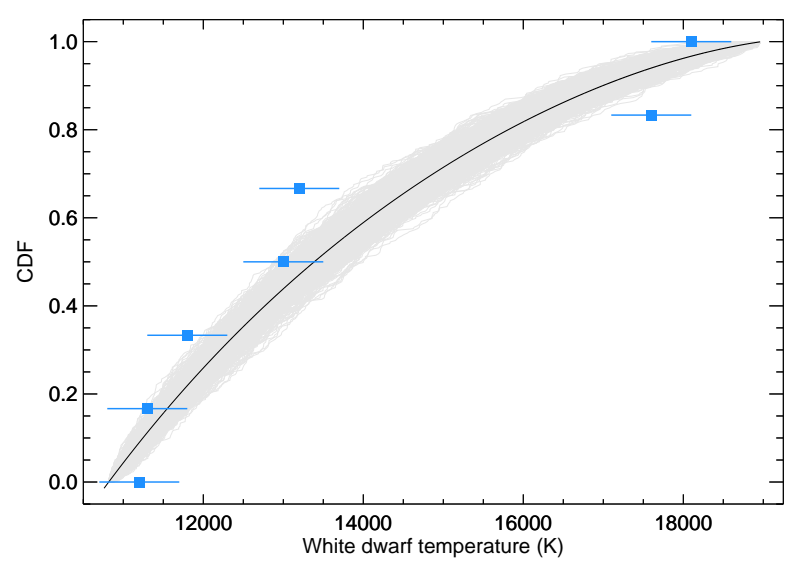

FIG. 6.- CDF of detected WD temperatures compared to $N$ body model predictions. The distribution from the $N$-body model of NGC 188 is shown as a black line (Geller et al.|2013). The light gray lines are from 1000 Monte Carlo bootstrap resamples of the $N$ body distribution. The seven WDs detected in this study are shown as solid blue squares at the temperatures given in Figures 2 and 3 with $500 \mathrm{~K}$ error bars. A KS test indicates the CDF of the detected WD temperatures is consistent with being drawn from the same parent population as the $N$-body model distribution $(p=0.97)$.

in NGC 188 at 7 Gyr, one of which formed from mass transfer. A separate $N$-body study of the open cluster M67 also fails to create a high fraction of mass transferformed BSSs as observed in NGC 188 (Hurley et al. 2005), although the progenitor binary period distributions used were not realistic.

The lack of BSSs in $N$-body models (Geller et al. 2013) may be attributed to an incomplete description of mass transfer processes in the binary population synthesis models used within the $N$-body code. Geller et al. (2013) utilize the NBODY6 code (Aarseth 2003), which relies similar algorithms to those implemented in the Binary-Stellar Evolution (BSE) code of Hurley et al. (2002) to track binary evolution.

Although there are too few BSSs, Geller et al. (2013) note that the model produces a large number of longperiod post-common envelope $(\mathrm{CE})$ binaries that are not observed in such frequency in NGC 188 or the field. The mass transfer parameterization in BSE may need to be adjusted such that these sources go through stable Roche lobe overflow rather than $\mathrm{CE}$. Converting the post-CE binaries to BSS+WD binaries would bring the total mass transfer BSS population to 10 systems at $7 \mathrm{Gyr}$, for a mass-transfer formation frequency of $67 \%$. This matches the inferred mass-transfer formation frequency of $67 \%$ measured in this study for the total NGC 188 BSS population.

However, if the parameterization of mass transfer is amended in BSE the consistency of the age distributions from the $N$-body model and and these results should be revisited.

Binary population synthesis models are important tools for theoretical astronomy that allow full- $N$ models of rich open clusters to run in a reasonable amount of time. We hope that observations such as these help constrain the parameterizations used so that population synthesis models can be as accurate as possible. 


\section{SUMMARY}

We utilize a FUV photometric study of the BSS binaries in the old open cluster NGC 188 to search for FUV excesses indicative of WD companions and thereby BSSs formed through mass transfer. We detect four BSSs with hot (temperatures greater than 12,000 K) WD companions. Since WDs cool as they age we use the estimated temperatures of the WD companions to place limits on the WD ages. These four systems are younger than $250 \mathrm{Myr}$, indicating the mass transfer in these binaries ended very recently. By further comparing the expected and observed FUV emission on a starby-star basis with a modeled Monte Carlo distribution of expected BSS emission, we detect three additional cool WD companions with temperatures between 11,000$12,000 \mathrm{~K}$.

In total, the HST data point to seven BSSs forming through mass transfer in the past $400 \mathrm{Myr}$. Analyzing the location of these recently-formed BSSs on an optical CMD reveals that these very young BSSs are both on and off the ZAMS. Thus, while BSSs near the ZAMS are likely among the youngest of the population, distance from the ZAMS is not a direct indicator of BSS age. Single-star isochrones may not be appropriate for determining the age of luminous BSSs. We do not find a clear separation in the optical CMD between mass transfer-formed BSSs and the non-velocity variable BSSs possibly formed through collisions or mergers of contact binaries.

The seven detected WDs in this study set a lower limit of $33 \%$ for the mass-transfer formation frequency of BSSs in NGC 188. In addition, we explore the possibility of other formation mechanisms by comparing overall properties of the BSS population to theoretical predictions. Due to the BSS binary properties it is very unlikely that any of the SB1 BSSs formed through dynamical collisions. Collisions typically create either single BSSs or BSSs with long periods that are not detectable with RVs (Geller et al. 2013). It is unlikely that any of the non-velocity variable BSSs formed through Case B or Case C mass transfer, as those mass transfer products are most often binaries with periods well within our RV sensitivity (Chen \& Han 2008; Geller et al. 2009). Current $N$-body models (Geller et al.|2013) predict that the Kozai mechanism produces 0.5 BSSs at the age of NGC 188, so we assume that one SB1 binary may have formed via merger in a hierarchical triple via the Kozai mechanism. As a result, we find that 14 of the 15
SB1 BSSs likely formed through mass transfer. This results in a total mass-transfer formation frequency of approximately 67\% (14 of 21) for BSSs in NGC 188. These results strongly support the previous predictions in Mathieu \& Geller (2009) and Geller \& Mathieu (2011) that the majority of the NGC 188 BSSs form through mass transfer.

The distribution of BSS ages found in this study is fully consistent with the mass transfer-formed BSS age distribution of mass-transfer formed BSSs in the $N$ body simulations of Geller et al. (2013). However, the frequency of mass-transfer formed BSSs found here challenges the results of $N$-body models that have difficulty creating numerous mass transfer BSSs (Hurley et al. 2005; Geller et al. 2013). It is possible that the parameterization of unstable mass transfer causes too many BSS-progenitor binaries to go into a stage of CE rather than stable Roche lobe overflow. If the parameterization within the $N$-body model of NGC 188 was adjusted such that the long-period CE systems instead went through stable mass transfer, the model would reproduce the BSS mass-transfer formation frequency measured in this study.

These BSS+WD binary detections represent some of the most well-characterized post-mass transfer systems to date, and provide an unparalleled opportunity to constrain detailed mass transfer and binary evolution models (Gosnell et al. 2014). These BSS+WD binaries will also help address the discrepancy between observations and $N$-body and population synthesis results.

We are grateful to the anonymous referee whose comments and suggestions improved this work. We thank K. Milliman for sharing the $\mathrm{H} \alpha$ BSS temperatures before publication. We are extremely grateful to $\mathrm{P}$. Bergeron for sharing his grid of WD atmosphere models. N. M. G. acknowledges support from the W. J. McDonald Postdoctoral Fellowship. R. D. M. and N. M. G. (while previously at UW-Madison) are supported through HST Program number 12492, provided by NASA through a grant from the Space Telescope Science Institute, which is operated by the Association of Universities for Research in Astronomy, Incorporated, under NASA contract NAS5-26555. A. S. is supported by the Natural Sciences and Engineering Research Council of Canada. A. M. G. is funded by a National Science Foundation Astronomy and Astrophysics Postdoctoral Fellowship under Award No. AST-1302765.

\section{REFERENCES}

Aarseth, S. J. 2003, Gravitational N-Body Simulations (Cambridge: Cambridge Univ. Press)

Abate, C., Pols, O. R., Izzard, R. G., Mohamed, S. S., \& de Mink, S. E. 2013, A\&A, 552, A26

Boffi, F. R., Sirianni, M., Lucas, R. A., Walborn, N. R., \& Proffitt, C. R. 2008, Technical Instrument Report ACS 2008-002

Burbidge, E. M., \& Sandage, A. 1958, ApJ, 128, 174

Carney, B. W., Latham, D. W., Laird, J. B., Grant, C. E., \& Morse, J. A. 2001, AJ, 122, 3419

Castelli, F., \& Kurucz, R. L. 2004, in IAU Symposium 210, Modelling of Stellar Atmospheres, ed. N. Piskunov et al., A20, arXiv:astro-ph/0405087

Chen, X., \& Han, Z. 2008, MNRAS, 387, 1416

Dieball, A., Knigge, C., Zurek, D. R., et al. 2005, ApJ, 634, L105
Feldman, P. D., Weaver, H. A., Saur, J., \& McGrath, M. A. 2010, Hubble after SM4. Preparing JWST

Ferraro, F. R., Fusi Pecci, F., Bellazzini, M., et al. 1995, A\&A, 294,80

Ferraro, F. R., Paltrinieri, B., Rood, R. T., \& Dorman, B. 1999, ApJ, 522, 983

Ferraro, F. R., D’Amico, N., Possenti, A., et al. 2001, ApJ, 561, 337

Ferraro, F. R., Sabbi, E., Gratton, R., et al. 2006, ApJ, 647, 53

Ferraro, F. R., Beccari, G., Dalessandro, E., et al. 2009, Nature, 462, 1028

Geller, A. M., Hurley, J. R., \& Mathieu, R. D. 2013, AJ, 145, 8

Geller, A. M., \& Mathieu, R. D. 2012, AJ, 144, 54

Geller, A. M., \& Mathieu, R. D. 2011, Nature, 478, 356 
Geller, A. M., Mathieu, R. D., Harris, H. C., \& McClure, R. D. 2009, AJ, 137, 3743

Geller, A. M., Mathieu, R. D., Harris, H. C., \& McClure, R. D. 2008, AJ, 135, 2264

Gonzaga, S., et al., 2013, ACS Data Handbook, Version 7.1 (Baltimore: STScI).

Gosnell, N. M., Mathieu, R. D., Geller, A. M., et al. 2014, ApJ, $783, \mathrm{~L} 8$

Henden, A., \& Munari, U. 2014, Contributions of the Astronomical Observatory Skalnate Pleso, 43, 518

Holberg, J. B., \& Bergeron, P. 2006, AJ, 132, 1221

Hurley, J. R., Tout, C. A., \& Pols, O. R. 2002, MNRAS, 329, 897

Hurley, J. R., Pols, O. R., Aarseth, S. J., \& Tout, C. A. 2005, MNRAS, 363, 293

Johnson, H. L., \& Sandage, A. R. 1955, ApJ, 121, 616

Knigge, C., Leigh, N., \& Sills, A. 2009, Nature, 457, 288

Knigge, C., Dieball, A., Maíz Apellániz, J., et al. 2008, ApJ, 683, 1006

Knigge, C., Gilliland, R. L., Dieball, A., et al. 2006, ApJ, 641, 281

Kroupa, P. 2001, MNRAS, 322, 231

Leigh, N., Sills, A., \& Knigge, C. 2007, ApJ, 661, 210

Leigh, N., \& Sills, A. 2011, MNRAS, 410, 2370

Leigh, N., Sills, A., \& Knigge, C. 2011, MNRAS, 416, 1410

Leigh, N., \& Geller, A. M. 2012, MNRAS, 425, 2369

Leigh, N., Knigge, C. Sills, A., et al. 2013, MNRAS, 428, 897

Leonard, P. J. T. 1989, AJ, 98, 217

Leonard, P. J. T. 1996, ApJ, 470, 521

Lombardi, J. C., Jr., Warren, J. S., Rasio, F. A., Sills, A., \& Warren, A. R. 2002, ApJ, 568, 939

Mapelli, M., Sigurdsson, S., Ferraro, F. R., et al. 2006, MNRAS, 373,361

Mapelli, M., Ripamonti, E., Tolstoy, E., et al. 2007, MNRAS, 380, 1127

Martin, D. C., Fanson, J., Schiminovich, D., et al. 2005, ApJ, 619, L1

Mathieu, R. D., \& Geller, A. M. 2014, in Ecology of Blue Stragglers, ed. H. Boffin, G. Carraro, \& G. Beccari, arXiv:1406.3467

Mathieu, R. D., \& Geller, A. M. 2009, Nature, 462, 1032

Mathieu, R. D. 2000, in ASP Conf. Ser. 198, Stellar Clusters and Associations: Convection, Rotation, and Dynamos, ed. R. Pallavicini, G. Micela, \& S. Sciortino (San Francisco, CA: ASP), 517
McCrea, W. H. 1964, MNRAS, 128, 147

Meibom, S., Grundahl, F., Clausen, J. V., et al. 2009, AJ, 137, 5086

Momany, Y., Held, E. V., Saviane, I., et al. 2007, A\&A, 468, 973

Naoz, S., \& Fabrycky, D. C. 2014, ApJ, 793, 137

Paczyński, B. 1971, ARA\&A, 9, 183

Perets, H. B., \& Fabrycky, D. C. 2009, ApJ, 697, 1048

Perets, H. B. 2014, in Ecology of Blue Stragglers, ed. H. Boffin, G. Carraro, \& G. Beccari, arXiv:1406.3490

Piotto, G., De Angeli, F., King, I., R., et al. 2004, ApJ, 604, L109

Platais, I., Kozhurina-Platais, V., Mathieu, R. D., Girard, T. M., \& van Altena, W. F. 2003, AJ, 126, 2922

Preston, G. W., \& Sneden, C. 2000, AJ, 120, 1014

Ramírez, I., \& Meléndez, J. 2005, ApJ, 626, 465

Rodríguez-Merino, L. H., Chavez, M., Bertone, E., \& Buzzoni, A. 2005, ApJ, 626, 411

Rozyczka, M., Kaluzny, J., Thompson, I. B., et al. 2013, AcA, 63, 67

Rucinski, S. M. 2000, AJ, 120, 319

Sandage, A. R. 1953, AJ, 58, 61

Sandquist, E. L. 2005, ApJ, 635, L73

Santana, F. A., Munoz, R. R., Geha, M., et al. 2013, ApJ, 774, 106

Sarajedini, A., von Hippel, T., Kozhurina-Platais, V., \&

Demarque, P. 1999, AJ, 118, 2894

Sills, A., Faber, J. A., Lombardi, J. C., Jr., Rasio, F. A., \& Warren, A. R. 2001, ApJ, 548, 323

Sills, A., Bailyn, C. D., Edmonds, P. D., \& Gilliland, R. L. 2000, ApJ, 535, 298

Sills, A., Lombardi, J. C., Jr., Bailyn, C. D., et al. 1997, ApJ, 487,290

Skrutskie, M. F., Cutri, R. M., Stiening, R., et al. 2006, AJ, 131, 1163

Talamantes, A., Sandquist, E. L., Clem, J. L., et al. 2010, AJ, 140,1268

Tian, B., Deng, L., Han, Z., \& Zhang, X. B. 2006, A\&A, 455, 247

Tremblay, P.-E., Bergeron, P., \& Gianninas, A. 2011, ApJ, 730, 128

Webbink, R. F. 1976, ApJ, 209, 829

Wood, M. A. 1995, LNP Vol. 443: White Dwarfs, 41 